\title{
58.3: Polarization-insensitive Liquid-Crystal Tunable Optical Filter
}

\author{
T. Kataoka, S. E. Day, D. R. Selviah, and A. Fernandez \\ Department of Electronic and Electrical Engineering, University College London, \\ Torrington Place, London, WC1E 7JE, UK \\ Correspondence Tel: ++44 (0) 7679 3056, Email: d.selviah@ee.ucl.ac.uk
}

\begin{abstract}
Liquid crystal filled Fabry-Perot filters (LC-FPF) can be used as tunable optical filters. The filters can be made insensitive to the incident polarization by using additional birefringent layers. An analysis of the polarization properties of these resonant structures is presented for two configurations.
\end{abstract}

\section{Introduction}

Liquid crystal Fabry-Perot filters (LC-FPF) have been proposed as electrically tunable optical wavelength filters for wavelength division multiplexing (WDM) systems in telecommunications [1, 2]. They are based on the structure of the Fabry-Perot étalon but the gap between a pair of highly reflective mirrors is filled with planar aligned liquid crystal (LC). The LC-FPF can be tuned by changing the refractive index of the LC electrically with an external electric field instead of the more common method of changing the physical distance between the two mirrors. The LCFPF is electrically tunable with a wide-tuning range [2] and can have a narrow-band response [3]. However, a disadvantage is that the LC-FPF is sensitive to the polarization state of the incident beam. This causes a fluctuation of the peak intensity when the input polarization state is varied. Single mode fiber used for optical fiber communication suffers from polarization mode dispersion, resulting in an unknown, unpredictably varying output polarization state. Therefore, ideal optical filters should be insensitive to the input polarization state.

When unpolarized beam travels through a conventional LC-FPF, it can be considered to be resolved into two components. One of them is parallel (the extraordinary ray) and the other (the ordinary ray) is perpendicular to the optic axis of the LC. Each polarization component gives rise to its own periodic array of resonant peaks in wavelength. The overall spectral response of the device can be considered to consist of the sum of these two spectral responses for unpolarized incident light. When an external electric field is applied across the LC layer inside the FPF, the resonant spectrum corresponding to the extraordinary refractive index can be tuned. However, the other resonant spectrum corresponding to the ordinary refractive index is not tuned and is fixed. This means that the LC-FPF can be tuned for only one polarization state, whose direction is parallel to the optic axis of the LC. Figure 1 shows our modeled result for the total transmitted intensity for a conventional LC-FPF with unpolarized light. The plot shows both the stationary set of resonant peaks and the variable set of peaks. If the input polarization varies between these polarizations, the transmission changes from one set of peaks to the other set, which causes a large intensity fluctuation. In order to ensure a spectrum which does not depend on the input polarization state, the two sets of orthogonal polarization peaks need to coincide and need to tune at the same rate when the electric field is varied.

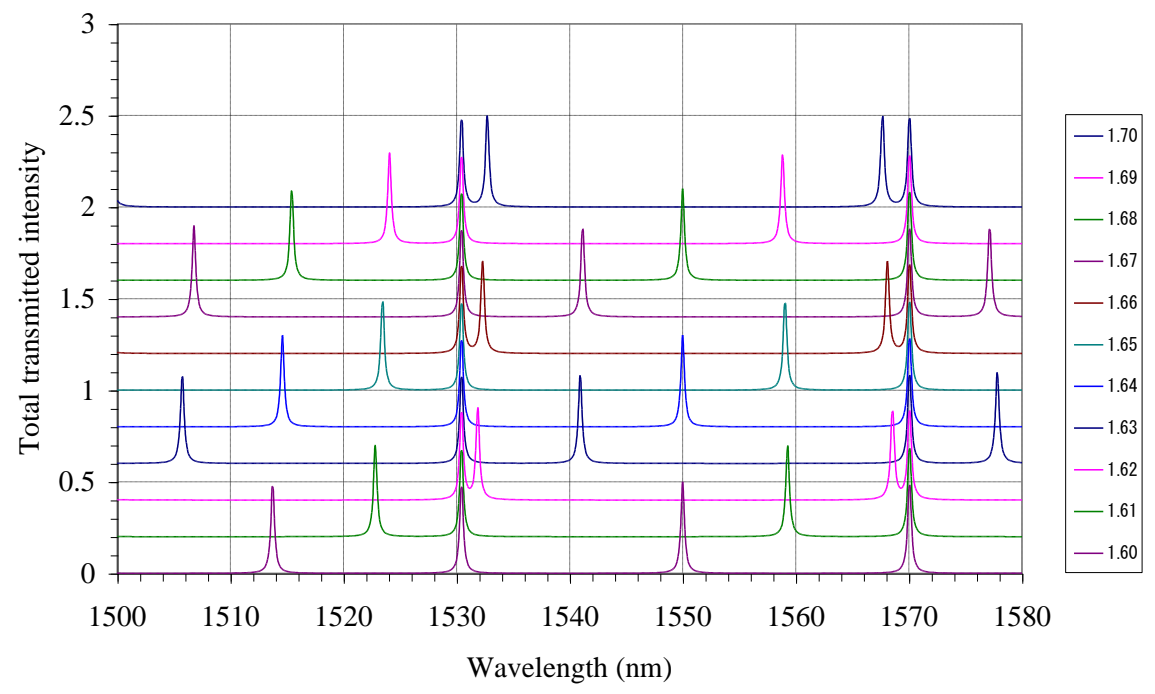

Figure 1. Transmittance spectra for different values of $L C$ birefringence for a conventional LC-FPF in case of unpolarized incident light, 9 dielectric mirrors layers,

LC layer (HWP, $n_{0}=1.50, n_{e}=1.70$, second-order) 
Some solutions for polarization sensitivity have been introduced and demonstrated:

1) The use of 'polarization diversity techniques' [4] which requires costly additional optical components.

2) The use of the twisted orientation for the LC [5], which gives small tuning range with high voltage.

3) The addition of extra birefringent quarter wave plates (QWP) into the cavity of the LC-FPF in order to change the polarization upon reflection within the cavity [6].

The work in this paper presents the results for modeling the LCFPF including first one and then two QWPs to change the polarization within the cavity and to keep the optical path for two polarized transmitted beams the same.

\section{Liquid-Crystal Fabry-Perot Filter with Birefringent Layers}

We have designed and investigated two types of LC-FPFs, whose LC is homogeneously aligned, with a single QWP or a pair of QWPs, by modeling with the 4 x 4 matrix Berreman method [7, 8]. We calculated the total transmitted intensity and output polarization state of the LC-FPFs.

\subsection{Liquid-Crystal Fabry-Perot Filter with a Single Quarter-Wave Plate}

The liquid crystal Fabry-Perot filter (LC-FPF) configuration with a single quarter-wave plate (QWP) is shown in Figure 2 (a). The optic axis of the single QWP is at $45^{\circ}$ to that of the liquid crystal (LC) layer. The ordinary (1.50) and the extraordinary (1.70) refractive indices of the QWP are chosen to be the same as those of the LC layer. The design wavelength for the QWPs is $1550 \mathrm{~nm}$. The optic axis of the LC layer is along the $\mathrm{X}$ axis, parallel to the $\mathrm{P}$ polarization in our model.

Figure 2 (a) shows how incident circularly polarized light is converted in a LC-FPF with a single QWP. When a left or right circularly polarized beam travels through the QWP, the polarization becomes either $\mathrm{P}$ or $\mathrm{S}$ polarized, depending on the sense of the circular polarization that was first incident. The $\mathrm{P}$ and $\mathrm{S}$ polarizations are parallel or perpendicular to the optic axis of the LC layer.

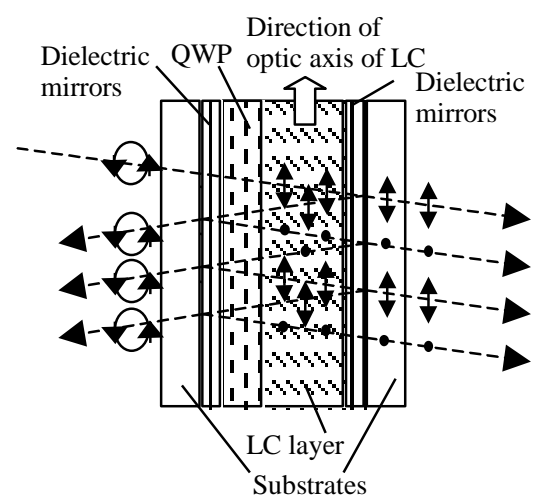

Figure 2 (a). Structure of LC-FPF with a single QWP in the case of incident circularly polarized light

Figure 3 shows our modeled results, for a LC-FPF with a single QWP with the extraordinary refractive index of the LC layer varied and with unpolarized incident beam. The resonant peaks can be observed and the free spectral range (FSR) is approximately $12 \mathrm{~nm}$. All of the resonant peaks shift to shorter wavelengths as the extraordinary refractive index is decreased.

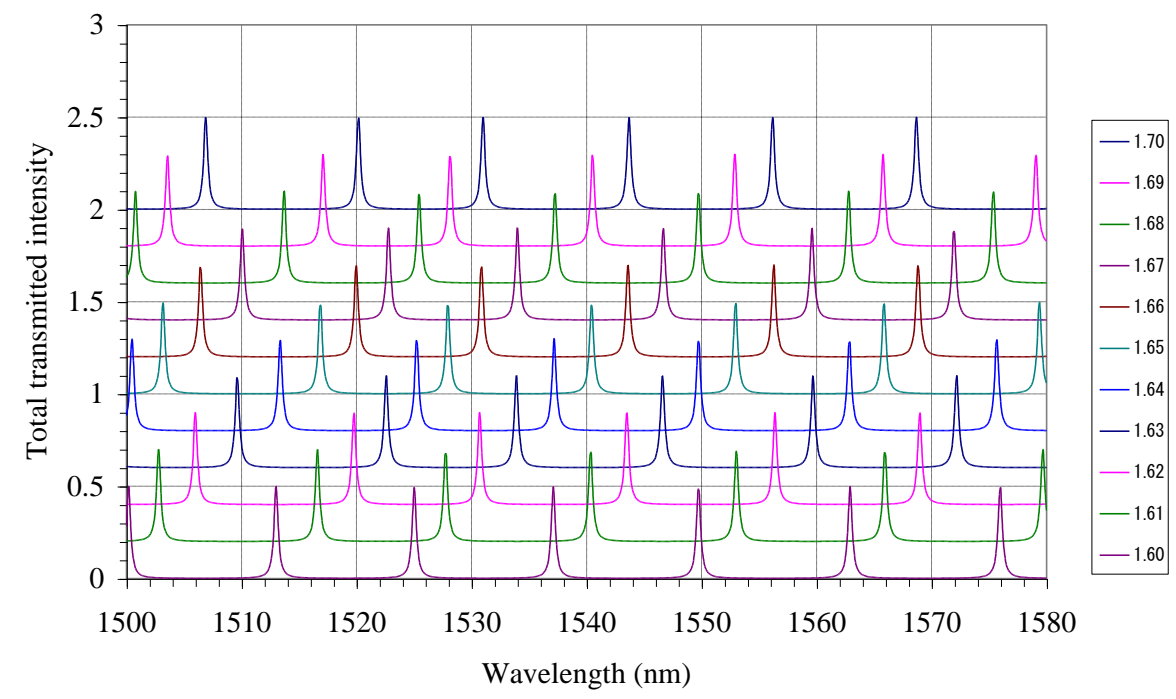

Figure 3. Transmission spectra for different values of LC birefringence for a LC-FPF with a single QWP in the cavity in case of unpolarized incident light, 9 dielectric mirror layers, QWP $\left(n_{0}=1.50, n_{e}=1.70\right.$, first-order $)$, LC layer $\left(H W P, n_{0}=1.50, n_{e}=1.70\right.$, second-order $)$ 
The polarization of each peak alternates between $45^{\circ}$ and $-45^{\circ}$ linear polarizations. The reason for this can be seen in Figure 2 (a) where the output light polarization after each double pass through the filter can be seen to alternate. Orthogonal polarizations cannot interfere and so the effective optical path for interference is four times the optical path through the filter, instead of the usual twice. This results in the reduced FSR and the alternating polarization for each peak. Therefore, this filter is suitable for use with a completely unpolarized light source where all of the fringes are required to tune. An example of an application might be as a spectrometer for a gas sensor.

\subsection{Liquid-Crystal Fabry-Perot Filter with a Pair of Quarter-Wave Plates}

Another configuration we have investigated is a LC-FPF where the LC layer is sandwiched between two QWPs. The optic axes of the QWPs are crossed and are at $+45^{\circ}$ and $-45^{\circ}$ to that of the optic axis of the planar aligned LC. Figure 2 (b) shows how circularly polarized light changes inside the LC-FPF. When a right or left circularly polarized beam travels through the first QWP of the LC-FPF, it is converted into a $\mathrm{P}$ or $\mathrm{S}$ linearly polarized beam, whose direction is parallel or perpendicular to the optic axis of LC layer. Then, it travels through the LC layer without changing polarization. The next QWP is in front of a mirror and so the effect of passing through the QWP twice upon reflection is that the plane polarized beam in the LC has the plane of polarization rotated by $90^{\circ}$. The beam then continues as $\mathrm{S}$ or $\mathrm{P}$ polarized light, where before it was $\mathrm{P}$ or $\mathrm{S}$ polarized. As a result, the optical path of right or left circularly polarized light is the same after passing twice through the cavity. The diagram shows the light that is transmitted through the partially reflecting dielectric mirror and it can be seen that the path difference between each of these beams is the regardless of whether it started as right or left circularly polarized. As a result, the interference should result in a transmission spectrum which is independent of the incident polarization.

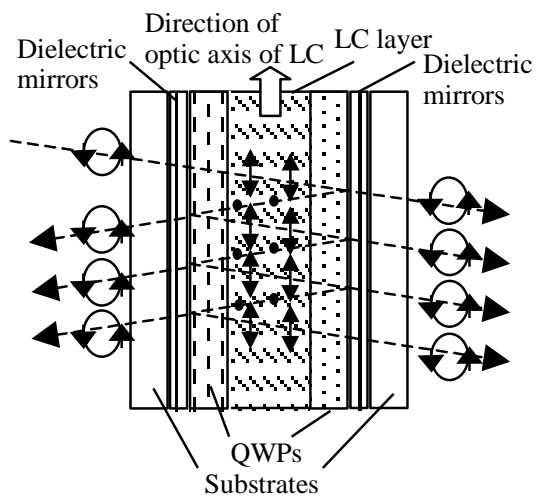

Figure 2 (b). Change of polarization in a LC-FPF having a pair of QWPs in the case of circularly incident polarized light

Once again modeled results are shown for different values of birefringence for the LC layer. Figure 4 shows the spectra for this configuration for a finesse of $\mathrm{F}=52$ and it can be seen that all peaks move to shorter wavelengths with the decrease of the extraordinary refractive index of the LC, but now the FSR corresponds to a double pass of the cavity. However, there is a slight splitting of some of the peaks. A polarization analysis of these peaks gives a better understanding of the origin of the splitting, which is necessary in order to reduce it.

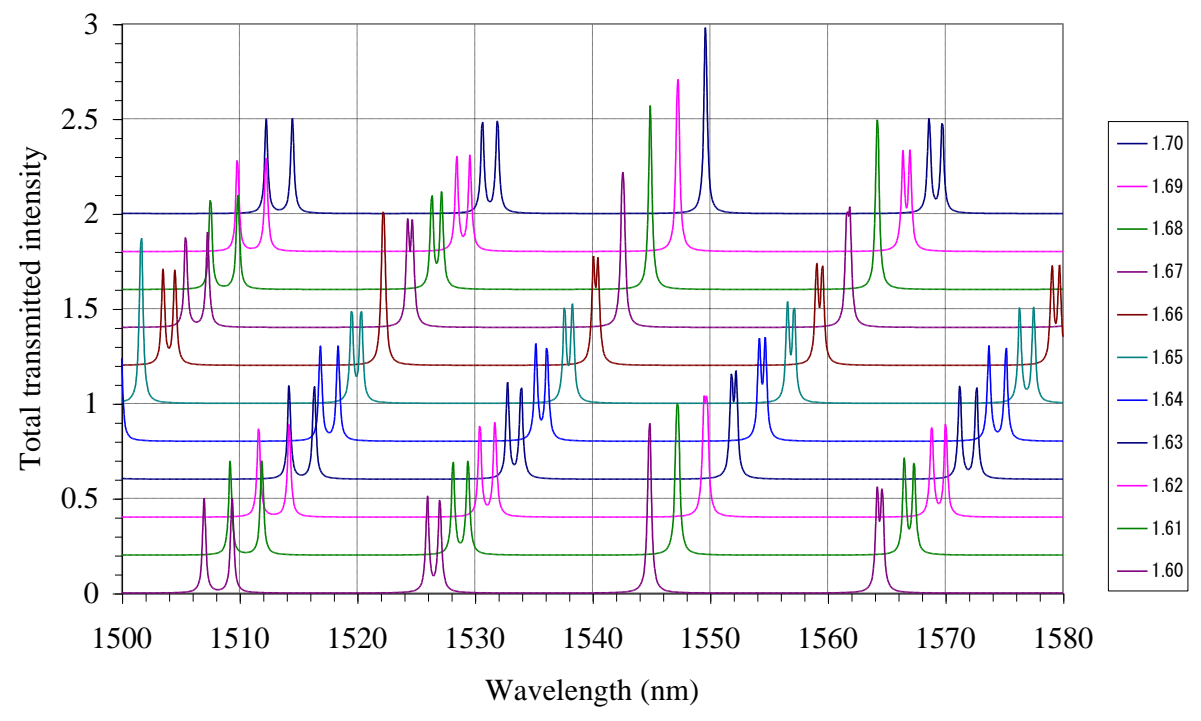

Figure 4. Transmission spectra for different values of LC birefringence of a LC-FPF with two QWPs in the cavity in the case of unpolarized incient light, 9 dielectric mirror layers, QWPs $\left(n_{0}=1.50, n_{e}=1.70\right.$, first-order $)$, LC layer $\left(H W P, n_{0}=1.50, n_{e}=1.70\right.$, second-order $)$ 


\section{Summary}

Previous theoretical work [6] reported the use of QWPs within the cavity of the LC-FPF in order to reduce the polarization sensitivity. Experimental work [9] confirmed the results and showed the splitting that is predicted (Figure 4). The present investigation into the origin of the splitting effect analyzes two different optical configurations and also includes detailed analysis of the polarization. Firstly, a LC-FPF with a single QWP shows that all peaks can be tuned with an external electric field. Therefore, the device with a single QWP can be used in systems where the input light is unpolarized. For example, in the application of spectrometers for gas sensors, unpolarized incident beam can be used as the light source and since the gases to be detected are not anisotropic, they will not alter the incident polarization. So, it is sufficient to monitor the change in absorption at a wavelength corresponding to a gas absorption line for any one polarization. Secondly, the LC-FPF with a pair of QWPs gives a degree of polarization insensitive operation, but for high Finesse filters there is some splitting of the transmitted peaks depending on the incident polarization. For some values of wavelength and birefringence the splitting is reduced. A better understanding of the origin of the splitting should lead to improved and optimized designs for polarization insensitive operation of the LC-FPF.

\section{Acknowledgements}

We would like to thank Kai Wang in the Optical Devices and Systems group at UCL for helpful discussions regarding Matlab computer programs. Our thanks to SID Information Display International Symposium for giving us the opportunity to present this paper.

\section{References}

[1] J. S. Patel, M. A. Saifi, D. W. Berreman, C. Lin, N. Andreadakis, and S. D. Lee: Appl. Phys. Lett, Vol. 57, No. 17, pp. 1718-1720 (1990)

[2] M. W. Maeda, J. S. Patel, C. Lin, J. Horrobin, and R. Spicer: IEEE Photon. Tech. Lett, Vol. 2, No. 11, pp. 820-822 (1990)

[3] K. Hirabayashi, H. Tsuda, and T. Kurokawa: IEEE Photon. Tech. Lett, Vol. 3, No. 3, pp. 213-215 (1991)

[4] J. S. Patel and M. W. Maeda: IEEE Photon. Tech. Lett, Vol. 3, No. 8, pp. 739-740 (1991)

[5] J. S. Patel and S. D. Lee: Appl. Phys. Lett, Vol. 58, No. 22, pp. 2491-2493 (1991)

[6] S. E. Day, D. R. Selviah, H. Manthopoulos, and M. Wiltshire: British Liquid Crystal Society Annual Conference, Exeter, 29-31st, March (1995)

[7] D. W. Berreman: J. Opt. Soc. Am, Vol. 62, No. 4, pp. 502$510(1972)$

[8] D. W. Berreman: J. Opt. Soc. Am, Vol. 63, No. 11, pp. 1374$1380(1973)$

[9] T. Morita and K. M. Johnson: Proc. SPIE Liquid Crystal 2, San Diego, pp. 152-162 (1998)

\section{Columns on Last Page Should Be Made As Close to Equal Length}

